Article

\title{
General Relativity with a Positive Cosmological Constant $\Lambda$ as a Gauge Theory
}

\author{
Marta Dudek ${ }^{1}$ and Janusz Garecki ${ }^{2, *}$ \\ 1 Institute of Mathematics, University of Szczecin, Wielkopolska 15, 70-451 Szczecin, Poland; \\ marta.dudek@vp.pl \\ 2 Institute of Mathematics and Cosmology Group, University of Szczecin, Wielkopolska 15, \\ 70-451 Szczecin, Poland \\ * Correspondence: janusz.garecki@usz.edu.pl
}

Received: 16 Ooctober 2018; Accepted: 4 February 2019; Published: 21 February 2019

check for updates

\begin{abstract}
In this paper, we show that the general relativity action (and Lagrangian) in recent Einstein-Palatini formulation is equivalent in four dimensions to the action (and Langrangian) of a gauge field. First, we briefly showcase the Einstein-Palatini (EP) action, and then we present how Einstein fields equations can be derived from it. In the next section, we study Einstein-Palatini action integral for general relativity with a positive cosmological constant $\Lambda$ in terms of the corrected curvature $\Omega_{\text {cor }}$. We see that in terms of $\Omega_{\text {cor }}$ this action takes the form typical for a gauge field. Finally, we give a geometrical interpretation of the corrected curvature $\Omega_{c o r}$.
\end{abstract}

Keywords: action integral; fiber bundle; connection in a principal fiber bundle and its curvature; pull-back of forms; Lie groups and their algebras

\section{Introduction: Einstein-Palatini Action for General Relativity}

In this section, we remind the author briefly about Einstein-Palatini formalism for general relativity (GR).

The Einstein field equations can be derived from postulation that the Einstein-Hilbert (EH) action is true action for GR. Albert Einstein firstly used only the metric as an independent variable to do variation of this action. The connection with EH action, in this approach, is the metric and symmetric Levi-Civita connection. Later, Einstein and Palatini proposed to take the metric and affine connection as independent variables in the action principle. This method allows computing the field equations for a more general metric affine connection rather than the Levi-Civita connection. Here, the spacetime admits torsion when matter Lagrangian is explicitly dependent on connection. Thus, the Einstein-Palatini formalism gives us a powerful tool for theories of gravitation that have more general Riemann-Cartan geometry.

In this section, we present Einstein-Palatini action in the recent formulation and how the Einstein fields equations can be computed from it.

The Einstein-Palatini action with cosmological constant $\Lambda$ in this new formulation see [1] is defined as follows

$$
S_{E P}=\frac{1}{4 \kappa} \int_{\mathcal{D}}\left(\vartheta^{i} \wedge \vartheta^{j} \wedge \Omega^{k l}+\frac{\Lambda}{6} \vartheta^{i} \wedge \vartheta^{j} \wedge \vartheta^{k} \wedge \vartheta^{l}\right) \eta_{i j k l}
$$

where $\Omega$ is the curvature of $\omega$ (spin connection) and $\kappa=8 \pi G / c^{4}$. All indices take values $(0,1,2,3)$. $\mathcal{D}$ means an established four-dimensional compact domain in spacetime. In the above formula, $\vartheta^{a}$ denotes 1-forms of the Lorentzian coreper such that the spacetime looks locally Minkowskian: $g=\eta_{i k} \vartheta^{i} \otimes \vartheta^{k}, \eta_{i k}=\operatorname{diag}(1,-1,-1,-1)$. 
$\eta_{i j k l}$ is a completely antisymmetric Levi-Civita pseudotensor: $\eta_{0123}=\sqrt{|g|}$, where $g:=\operatorname{det}\left(g_{i k}\right)$. In a Lorentzian coreper, $|g|=1$. Spin connection $\omega$ is a general metric connection (or Levi-Civita connection) in Lorentzian coreper. Strictly speaking, by spin connection, relativists understand the mixed components $\omega_{b \mu}^{a}$ of the connection $\omega$. These mixed components are defined in the following way. Firstly, we decompose connection form $\omega^{i}{ }_{k}$ in terms of the Lorentzian 1-forms $\vartheta^{l}$

$$
\omega_{k}^{i}=\omega_{k l}^{i} \vartheta^{l}
$$

Then, the decomposition of the forms $\vartheta^{l}$ in the natural cobasis $d x^{\mu}$

$$
\vartheta^{l}=h_{\mu}^{l} d x^{\mu}
$$

leads to

$$
\omega^{i}{ }_{k}=\omega_{k l}^{i} h_{\mu}^{l} d x^{\mu}=: \omega_{k \mu}^{i} d x^{\mu} .
$$

Relativists called the mixed components $\omega_{k \mu}^{i}$ the spin connection.

For the geometrical units $G=c=1$, Equation (1) takes the following form if $\epsilon$ and $\Lambda>0$

$$
S_{E P}=\frac{1}{32 \pi} \int_{\mathcal{D}}\left(\eta_{i j k l} \vartheta^{i} \wedge \vartheta^{j} \wedge \Omega^{k l}+\frac{\Lambda}{6} \eta_{i j k l} \vartheta^{i} \wedge \vartheta^{j} \wedge \vartheta^{k} \wedge \vartheta^{l}\right) \text {. }
$$

Adding to the geometric part $S_{E P}$ the matter action

$$
S_{m}=\int_{\mathcal{D}} L_{m a t}\left(\phi^{A}, D \phi^{A}, \vartheta^{i}\right),
$$

where $\phi^{A}$ means tensor-valued matter form and $D \phi^{A}$ its absolute exterior derivative, we obtain full action

$$
\begin{aligned}
S & =S_{E P}+S_{m} \\
& =\frac{1}{32 \pi} \int_{\mathcal{D}}\left(\eta_{i j k l} \vartheta^{i} \wedge \vartheta^{j} \wedge \Omega^{k l}+\frac{\Lambda}{6} \eta_{i j k l} \vartheta^{i} \wedge \vartheta^{j} \wedge \vartheta^{k} \wedge \vartheta^{l}\right)+\int_{\mathcal{D}} L_{m a t}\left(\phi^{A}, D \phi^{A}, \vartheta^{i}\right)
\end{aligned}
$$

After some calculations, one gets that the variation $\delta S=\delta S_{E P}+\delta S_{m}$ with respect to $\vartheta^{i}, \omega_{j}^{i}$ and $\phi^{A}$ reads

$$
\begin{aligned}
\delta S & =\int_{\mathcal{D}}\left[\frac{1}{8 \pi} \delta \vartheta^{i} \wedge\left(\frac{1}{2} \Omega^{k l} \wedge \eta_{k l i}+\Lambda \eta_{i}+8 \pi t_{i}\right)\right. \\
& \left.+\frac{1}{2} \delta \omega^{i}{ }_{j} \wedge\left(\frac{1}{8 \pi} D \eta_{i}{ }^{j}+s_{i}^{j}\right)+\delta \phi^{A} \wedge L_{A}+\text { an exact form }\right] .
\end{aligned}
$$

The three-forms: energy-momentum $t_{i}$, classical spin $s_{i}{ }^{j}$ and $L^{A}$ are defined by the following form of the variation $\delta L_{m}$

$$
\delta L_{m}=\delta \vartheta^{i} \wedge t_{i}+\frac{1}{2} \delta \omega_{j}^{i} \wedge s_{i}^{j}+\delta \phi^{A} \wedge L^{A}+\text { an exact form } .
$$

$\eta_{k l i}, \eta_{i}^{j}, \eta_{i}$ mean the forms introduced in the past by Trautman [2] (see also Appendix A).

The variations $\delta \vartheta^{i}, \delta \omega^{i}$ and $\delta \phi^{A}$ are vanishing on the boundary $\partial \mathcal{D}$ of the compact domain $\mathcal{D}$. 
Einstein's equations, similar to all other physical field equations, can be obtained from the variational principle, which is called the principle of stationary action or Hamiltonian principle. In our case, it has the following form:

$$
\delta S=0
$$

It leads us to the following sets of the field equations:

$$
\begin{aligned}
& \frac{1}{2} \Omega^{k l} \wedge \eta_{k l i}+\Lambda \eta_{i}=-8 \pi t_{i} \\
& D \eta_{i}{ }^{j}=\eta_{i k}{ }_{k} \wedge \Theta^{k}=-8 \pi s_{i}{ }^{j}
\end{aligned}
$$

and

$$
L_{A}=0 .
$$

$L_{A}=0$ represents equations of motion for matter field. These equations are not intrinsic in further our considerations, thus we omit them. We are interested only in the gravitational field equations, which are given by Equations (8) and (9). Equations (8)-(10) (but with $\lambda=0$ ) were first obtained by Trautman [2]. There is only one difference in our approach-the opposite sign of the spin tensor. Here $\Theta$ is the 2-form of torsion.

In vacuum where $t_{i}=s_{i}^{j}=0 \Longrightarrow \eta_{i}{ }_{k} \wedge \Theta^{k}=0 \Longrightarrow \Theta^{k}=0$, i.e., vanishing torsion, we get the standard vacuum Einstein's equations (EE) with cosmological constant $\Lambda$ and pseudoriemannian geometry

$$
\frac{1}{2} \Omega^{k l} \wedge \eta_{k l i}+\Lambda \eta_{i}=0
$$

In general, we have the Einstein-Cartan equations and Riemann-Cartan geometry (a metric geometry with torsion).

We also obtain the standard GR if we put $\frac{\delta L_{m}}{\delta \omega^{i}{ }_{k}}=0 \Longrightarrow s_{i}{ }^{k}=0 \Longrightarrow \eta_{i}{ }^{k} \wedge \Theta^{j}=0 \Longrightarrow \Theta^{j}=0$. It is GR inside spinless matter with equations

$$
\frac{1}{2} \Omega^{k l} \wedge \eta_{k l i}+\Lambda \eta_{i}=-8 \pi t_{i}
$$

One can show that $\frac{1}{2} \Omega^{k l} \wedge \eta_{k l i}=-G_{i}^{S} \eta_{s}$, where the Einstein tensor $G_{i}^{s}$ is defined as follows

$$
G_{i}^{s}=R_{i}^{s}-\frac{1}{2} \delta_{i}^{s} R
$$

Putting $t_{i}=T_{i}^{s} \eta_{s}$, we get from Equation (12)

$$
-G_{i}^{s} \eta_{s}+\Lambda \delta_{i}^{s} \eta_{s}=-8 \pi T_{i}^{s} \eta_{s}
$$

or

$$
G_{i}^{s}-\Lambda \delta_{i}^{s}=8 \pi T_{i}^{s}
$$

Equation (15) represents the standard Einstein equations with cosmological constant $\Lambda$ in tensorial notation with symmetric matter tensor: $T^{i k}=T^{k i}$. 
2. Results: Einstein-Palatini Action Integral for General Relativity in Vacuum and with Positive Cosmological Constant $\Lambda$ As Integral Action for a Gauge Field

Let us return to the Einstein-Palatini action (Equation(1)) in vacuum and introduce the duality operator $\star$ see Wise [3]

$$
\star:=-\frac{\eta_{i j k l}}{2} \Longrightarrow \eta_{i j k l}=-2 \star .
$$

Then, one has

$$
\begin{array}{r}
\eta_{i j k l} \Omega^{k l}=-2 \star \Omega_{i j}, \\
\eta_{i j k l} \vartheta^{k} \wedge \vartheta^{l}=-2 \star\left(\vartheta_{i} \wedge \vartheta_{j}\right)
\end{array}
$$

and the Einstein-Palatini action has the following form

$$
\begin{aligned}
S_{E P} & =-\frac{1}{2 \kappa} \int_{\mathcal{D}}\left(\vartheta^{i} \wedge \vartheta^{j} \wedge \star \Omega_{i j}+\frac{\Lambda}{6} \vartheta^{i} \wedge \vartheta^{j} \wedge \star\left(\vartheta_{i} \wedge \vartheta_{j}\right)\right) \\
& =-\frac{1}{2 \kappa} \int_{\mathcal{D}} \operatorname{tr}\left(\vartheta \wedge \vartheta \wedge \star \Omega+\frac{\Lambda}{6} \vartheta \wedge \vartheta \wedge \star(\vartheta \wedge \vartheta)\right) .
\end{aligned}
$$

Now, let us introduce the corrected curvature $\Omega_{\text {cor }}$

$$
\Omega_{c o r}:=\Omega+\frac{\Lambda}{3} \vartheta \wedge \vartheta \quad \Longrightarrow \vartheta \wedge \vartheta=-\frac{3}{\Lambda}\left(\Omega-\Omega_{\text {cor }}\right) .
$$

Substituting the last formula into Einstein-Palatini action, we get

$$
\begin{aligned}
S_{E P} & =\frac{1}{2 \kappa} \int_{\mathcal{D}} \operatorname{tr}\left(\vartheta \wedge \vartheta \wedge \star \Omega+\frac{\Lambda}{6} \vartheta \wedge \vartheta \wedge \star(\vartheta \wedge \vartheta)\right) \\
& =\frac{1}{2 \kappa} \int_{\mathcal{D}} \operatorname{tr}\left[\frac{3}{\Lambda}\left(\Omega-\Omega_{\text {cor }}\right) \wedge \star \Omega-\frac{\Lambda}{6} \frac{9}{\Lambda^{2}}\left(\Omega-\Omega_{\text {cor }}\right) \wedge \star\left(\Omega-\Omega_{\text {cor }}\right)\right] \\
& =\frac{3}{4 \Lambda \kappa} \int_{\mathcal{D}} \operatorname{tr}\left(2\left(\Omega-\Omega_{\text {cor }}\right) \wedge \star \Omega-\left(\Omega-\Omega_{\text {cor }}\right) \wedge \star\left(\Omega-\Omega_{\text {cor }}\right)\right) \\
& =\frac{3}{4 \Lambda \kappa} \int_{\mathcal{D}} \operatorname{tr}\left[2 \Omega \wedge \star \Omega-2 \Omega_{\text {cor }} \wedge \star \Omega-\Omega \wedge \star \Omega+\Omega_{\text {cor }} \wedge \star \Omega+\Omega \wedge \star \Omega_{\text {cor }}-\Omega_{\text {cor }} \wedge \star \Omega_{\text {cor }}\right] \\
& =\frac{3}{4 \Lambda \kappa} \int_{\mathcal{D}} \operatorname{tr}\left[\Omega \wedge \star \Omega-\Omega_{\text {cor }} \wedge \star \Omega+\Omega \wedge \star \Omega_{\text {Cor }}-\Omega_{\text {cor }} \wedge \star \Omega_{\text {cor }}\right]
\end{aligned}
$$

Because $-\Omega_{\text {cor }} \wedge \star \Omega+\Omega \wedge \star \Omega_{\text {cor }}$ reduces, we finally have

$$
S_{E P}=\frac{3}{4 \Lambda \kappa} \int_{\mathcal{D}} \operatorname{tr}\left[\Omega \wedge \star \Omega-\Omega_{\text {cor }} \wedge \star \Omega_{\text {cor }}\right] .
$$

The expression $\operatorname{tr}(\Omega \wedge \star \Omega)=\eta_{i j k l} \Omega^{i j} \wedge \Omega^{k l}$ is in four dimensions a topological invariant called Euler's form, which does not influence the equations of motion see Hayashi, Shirafuji [4]. Hence, in four dimensions, the Einstein-Palatini action is equivalent to

$$
S_{E P}=-\frac{3}{4 \Lambda \kappa} \int_{\mathcal{D}} \operatorname{tr}\left(\Omega_{c o r} \wedge \star \Omega_{c o r}\right)
$$


We see that the Einstein-Palatini action in four dimensions is effectively the quadratic function of the corrected Riemannian curvature, i.e., it has a form of the action for a gauge field.

The only difference is that in Equation (23) we have the star operator $\star$, which is different from Hodge star operator. Namely, our star operator acts onto"interior" indices (tetrad indices), not onto forms as Hode duality operator does.

The gauge group for the theory with the action in Equation (23) is the Lorentz group $\mathcal{L}=S O(1,3)$ or its double cover $S L(2, \mathbb{C})$.

It is interesting that $\Omega_{\text {cor }}=0$ for the de Sitter spacetime, which is the fundamental vacuum solution to the Einstein equations

$$
G_{i}^{s}-\Lambda \delta_{i}^{s}=0
$$

We would like to emphasize that in the case $\Lambda=0$ the above trick with $\Omega_{\text {cor }}$ breaks. Namely, we have in this case (see Section 3) $\Omega_{\text {cor }}=\Omega$ because $\left[e_{i}, e_{k}\right]=0$. This result formally trivializes $S_{E-P}$ action to the strange form $S_{E-P}=0$. Thsi is easily seen from Equation(21) or Equation(22). In the case $\Lambda<0$, one obtains the result analogical to Equation (23) with $\Omega_{c o r}=\Omega+\frac{\Lambda}{3} \vartheta \wedge \vartheta$ but this time $\Lambda<0$. We do not consider this case because it requires introducing the anti-de Sitter spacetime (and its isometry group $\mathrm{SO}(2,3)$ ) into calculations, which has very strange casual properties.

\section{Discussion: Geometrical Interpretation of the Corrected Curvature $\Omega_{\text {cor }}$}

Let $P\left(M_{4}, G d S\right)$ denote the principal bundle of de Sitter basis over a manifold $M_{4}$ (spacetime) with de Sitter group $(G d S)$ see Drechsler, Mayer [5], Gürsey [6] as a structure group. This group is isomorphic to the group $S O(1,4)$ see Drechsler, Mayer [5], Randono [1], Gürsey [6]. Let $\widetilde{\omega}$ be 1-form of connection in the principle fibre bundle $P\left(M_{4}, G d S\right)$. The form $\widetilde{\omega}$ has values in the algebra $\mathfrak{g}$ of the group $G d S$. This algebra splits (as a vector space) into direct sum

$$
\mathfrak{g}=\operatorname{so}(1,3) \oplus R^{(1,3)} .
$$

so $(3,1)$ denotes here algebra of the group $\mathrm{SO}(1,3)$, which is isomorphic to Lorentz group $\mathcal{L}$, and $R^{(1,3)}$ is a four-dimensional vector space of generalized translations (translations in the curved de Sitter spacetime). One can identify the de Sitter spacetime with the quotient $\mathrm{SO}(1,4) / \mathrm{SO}(1,3)$.

Let us define $s o(1,3)=: \mathfrak{h}, R^{1,3}=: \mathfrak{p}$. Then, we have see Wise $[3,7]$

$$
\mathfrak{g}=\mathfrak{h} \oplus \mathfrak{p},
$$

and

$$
[\mathfrak{h}, \mathfrak{h}] \subset \mathfrak{h},[\mathfrak{h}, \mathfrak{p}] \subset \mathfrak{p},[\mathfrak{p}, \mathfrak{p}] \subset \mathfrak{h}
$$

This means that the Lie algebra $\mathfrak{g}$ is a symmetric Lie algebra see Wise [3,7].

On the other hand, the spaces which satisfy Equations (26) and (27) are called globally symmetric Riemannian spaces see Drechsler [5].

Let $P\left(M_{4}, \mathcal{L}\right)$ denote the principal bundle of Lorentz basis over the manifold $M_{4}$. There exists a morphism of principal bundles

$$
f: P\left(M_{4}, \mathcal{L}\right) \longrightarrow P\left(M_{4}, G d S\right)
$$

analogical to the morphism of the bundle linear frames and the bundle affine frames see Kobayashi, Nomizu [8]. This morphism creates pull-back $f_{*} \widetilde{\omega}$ of the form $\widetilde{\omega}$ onto the bundle $P\left(M_{4}, \mathcal{L}\right)$. Here, $\widetilde{\omega}$ is the connection 1-form in the bundle $P\left(M_{4}, G d S\right)$. 
Let us denote this pull-back by $A$. $A$ is a 1 -form on $P\left(M_{4}, \mathcal{L}\right)$ with values in the direct sum see Kobayashi, Nomizu [8]

$$
\text { so }(1,3) \oplus R^{(1,3)} \text {. }
$$

Hence, we have a natural decomposition see Kobayashi, Nomizu [8]

$$
A=f_{*} \widetilde{\omega}=\omega+\theta,
$$

where $\omega$ is a 1 -form on $P\left(M_{4}, \mathcal{L}\right)$ with values in the algebra so(1,3) and $\theta$ is a 1 -form on $P\left(M_{4}, \mathcal{L}\right)$ with values on $R^{(1,3)}$. $\omega$ is a connection on the bundle $P\left(M_{4}, \mathcal{L}\right)$.

On the base $M_{4}$, the 1-form $\theta$ can be identified with 1-form $\vartheta$ already used in this paper: $\theta=\vartheta$. In the following, we work on the base space $M_{4}$ and write Equation (30) in the form

$$
A=\omega+\vartheta
$$

Let us compute a 2-form curvature $\widetilde{\Omega}$ of the pulled back $A$. From the definition, we have

$$
\begin{aligned}
\widetilde{\Omega} & =d A+\frac{1}{2}[A, A] \\
& =d(\omega+\vartheta)+\frac{1}{2}[\omega+\vartheta, \omega+\vartheta] \\
& =d \omega+\frac{1}{2}[\omega, \omega]+d \vartheta+\frac{1}{2}[\omega, \vartheta]+\frac{1}{2}[\vartheta, \omega]+\frac{1}{2}[\vartheta, \vartheta] .
\end{aligned}
$$

We introduce to our equations bases $\widetilde{M}_{i k}=-\widetilde{M}_{k i}$ of algebra so(1,3) and $e_{i}$ of vector space $R^{(1,3)}$. On these bases, we have

$$
\omega=\omega^{i}{ }_{k} \widetilde{M}_{i}^{k}=\omega^{i k} \tilde{M}_{i k}, \vartheta=\vartheta^{i} e_{i}
$$

$\left(\widetilde{M}_{i k}, e_{l}\right)$ forms the algebra of the de Sitter group (the basis of algebra $\left.\mathfrak{g}\right)$. Our elements $\widetilde{M}_{i k}=-\widetilde{M}_{k i}$ are real and connected with elements $M_{i k}=M_{k i}$ used see Drechsler, Mayer [5] in the following way

$$
\widetilde{M}_{i k}=\frac{i}{2} M_{i k} \Rightarrow M_{i k}=-2 i \widetilde{M}_{i k}
$$

The commutation relations for the algebra $s o(1,4)=s o(1,3) \oplus R^{(1,3)}$, in the terms of the elements $\left[\tilde{M}_{i k}, e_{l}\right]$, read

$$
\begin{aligned}
& {\left[\widetilde{M}_{i j}, \widetilde{M}_{k l}\right]=\frac{1}{2}\left(\eta_{i l} \widetilde{M}_{j k}+\eta_{j k} \widetilde{M}_{i l}-\eta_{i k} \widetilde{M}_{j l}-\eta_{j l} \widetilde{M}_{i k}\right)} \\
& {\left[e_{i}, \widetilde{M}_{j k}\right]=\frac{1}{2}\left(\eta_{i j} e_{k}-\eta_{i k} e_{j}\right)} \\
& {\left[e_{i}, e_{j}\right]=\frac{2 \widetilde{M}_{i j}}{R^{2}}}
\end{aligned}
$$

The following commutation relations are important in the further considerations see Gürsey [6], Dubničkova [9], Drechsler, Mayer [5].

$$
\begin{aligned}
& {\left[\widetilde{M}_{k i}, e_{l}\right]=\frac{1}{2}\left(\eta_{i l} e_{k}-\eta_{k l} e_{i}\right)} \\
& {\left[e_{i}, e_{k}\right]=\frac{2 \widetilde{M}_{i k}}{R^{2}}}
\end{aligned}
$$


where $R$ is the radius of the de Sitter spacetime. This radius $R$ is connected with $\Lambda$ by the formula $\Lambda=\frac{3}{R^{2}}$. Using the above equations, we have

$$
\widetilde{\Omega}=\Omega_{\omega}+\frac{1}{2} \omega_{k}^{i} \wedge \vartheta^{l}\left[\tilde{M}_{i}^{k}, e_{l}\right]+\frac{1}{2} \vartheta^{l} \wedge \omega_{k}^{i}\left[e_{l}, \tilde{M}_{i}^{k}\right]+\frac{1}{2} \vartheta^{i} \wedge \vartheta^{k}\left[e_{i}, e_{k}\right]+d \vartheta^{i} e_{i}
$$

where $\Omega_{\omega}=d \omega+\frac{1}{2}[\omega, \omega]$ is the curvature 2-form of the connection's $\omega$. Taking into consideration the commutation relations in algebra $\mathfrak{g}$ given by Equations (37) and (38), we obtain

$$
\begin{aligned}
\widetilde{\Omega} & =\Omega_{\omega}+\omega_{k}^{i} \wedge \vartheta^{l}\left[\widetilde{M}_{i}^{k}, e_{l}\right]+\vartheta^{i} \wedge \vartheta^{k} \frac{\widetilde{M}_{i k}}{R^{2}}+d \vartheta^{i} e_{i} \\
& =\Omega_{\omega}^{i k} \widetilde{M}_{i k}+\left(\omega_{. k}^{i} \wedge \vartheta^{k}\right) e_{i}+d\left(\vartheta^{i}\right) e_{i}+\frac{\vartheta^{i} \wedge \vartheta^{k} \tilde{M}_{i k}}{R^{2}} \\
& =\Omega_{\omega}^{i k} \tilde{M}_{i k}+\frac{\vartheta^{i} \wedge \vartheta^{k} \tilde{M}_{i k}}{R^{2}}+\left(d \vartheta^{i}+\omega_{. k}^{i} \wedge \vartheta^{k}\right) e_{i} \\
& =\Omega_{c o r r}^{i k} \tilde{M}_{i k}+\left(\mathcal{D}_{\rightarrow} \vartheta^{i}\right) e_{i} \\
& =\Omega_{c o r r}^{i k} \tilde{M}_{i k}+\Theta^{i} e_{i}
\end{aligned}
$$

$\Omega_{\text {cor }}:=\Omega_{\omega}+\frac{\vartheta \wedge \vartheta}{R^{2}}=\Omega_{\omega}+\frac{\Lambda}{3} \vartheta \wedge \vartheta$ and it denotes the corrected curvature of the connection $\omega$ on the bundle $P\left(M_{4}, \mathcal{L}\right)$ and $\Theta=\mathcal{D}_{\omega} \vartheta$ is a torsion of the connection $\omega$.

If we adjust the connection $\widetilde{\omega}$ in such a way that the connection $\omega$ is torsionless $(\Theta=0)$, i.e., if $\omega$ is Levi-Civita connection, then we get (after leaving the basis $s o(1,3)$ and $R^{(1,3)}$ )

$$
\widetilde{\Omega}=\Omega_{\omega}+\frac{\Lambda}{3} \vartheta \wedge \vartheta=\Omega_{c o r}
$$

In Section 2, we give the definition of the corrected curvature $\Omega_{\text {cor }}$ as follows:

$$
\Omega_{c o r}:=\Omega+\frac{\Lambda}{3} \vartheta \wedge \vartheta
$$

As one can see, this curvature is a curvature of the connection

$$
A:=f_{*} \widetilde{\omega}=\omega+\vartheta
$$

if $\Theta=0$, e.g., in Einstein-Cartan vacuum.

If $\Theta \neq 0$, e.g., in Einstein theory with spinning sources, then $\Omega_{\text {cor }}$ is the so(1,3)-part of the curvature $\widetilde{\Omega}$.

\section{Conclusions}

In this article, we have shown that in four dimensions the action integral for GR with a positive cosmological constant $\Lambda$ can be written in an analogical form to the form of the action integral for the typical gauge field. However, there is one difference - the star. Instead of the Hodge star, we have a slightly different star called the duality operator see Hayashi, Shirafuji [4], Wise [7].

Our result is important because it shows that there is no need to generalize GR and construct very complicated gravitational theories to obtain a gravitational theory as a gauge theory. The ordinary GR formulated in terms of tetrads and spin connection with cosmological constant $\Lambda>0$ is already a gauge theory with gauge group $\mathcal{L}=S O(1,3)$ or its double cover $S L(2, \mathbb{C})$. This fact is very interesting in connection with universality of the Einstein theory: every alternative metric theory of gravity can be reformulated as Einstein theory with additional "egzotic" matter fields see Kijowski [10], Schweizer [11]. Therefore, we present a following conjecture. 


\section{Conjecture:}

After the above reformulation, one can put the pure geometric part of the action (identical to the geometric part $S_{E P}$ ) for any alternative theory with $\Lambda>0$ in the form of Equation (23). This Conjecture will be studied in the future.

Some scientists see Wise [3,7], Randono [1] were concerned with this problem and they came to similar conclusions as ours, but they applied in their works the Cartan's approach to the connection in the principal bundle see Wise [7], Drechsler, Mayer [5], Sharpe [12]. This approach is not well known among geometrists and relativists. We have used only the standard theory of connection in the principal bundle which was created by Ehresmann and Cartan's student see Kobayashi, Nomizu [8], Gancarzewicz [13]. His approach is commonly used in differential geometry and in relativity. We would like to emphasize that the formulation of the EP action in the form of Equation (23) can be important for quantizing of general relativity (because gauge fields can be successfully quantized).

Author Contributions: M.D. and J.G. contributed equally to this work.

Funding: This research received no external funding.

Conflicts of Interest: The authors declare no conflict of interest.

\section{Appendix A}

$\eta$ forms and operations with them see [2]

Following Trautman [2], we define

$$
\eta_{i j k l}=\sqrt{|g|} \epsilon_{i j k l}
$$

where $\epsilon_{i j k l}$ is a Levi-Civita pseudotensor with properties

$$
\epsilon_{i j k l}=\left\{\begin{array}{cl}
1 & \begin{array}{l}
\text { if the sequence of indices } \mathrm{ijkl} \text { is an even permutation } \\
\text { of the sequence } 0,1,2,3
\end{array} \\
-1 & \begin{array}{l}
\text { if it is an odd permutation; and } \\
0
\end{array} \\
\text { if the sequence of indices ijkl is not an even permutation } \\
\text { of the sequence } 0,1,2,3
\end{array} .\right.
$$

and we take $\eta_{0123}=\sqrt{|g|}$. In Lorentzian coreper $|g|=1$.

One has see Trautman [2]:

$$
\begin{aligned}
& \eta_{i j k}=\vartheta^{l} \wedge \eta_{i j k l} \\
& \eta_{i j}=\frac{1}{2} \vartheta^{k} \wedge \eta_{i j k} \\
& \eta_{i}=\frac{1}{3} \vartheta^{j} \wedge \eta_{i j} \\
& \eta=\frac{1}{4} \vartheta^{i} \wedge \eta_{i} \\
& \vartheta^{n} \eta_{i j k l}=\delta_{l}^{n} \eta_{i j n}+\delta_{j}^{n} \eta_{l i k}-\delta_{i}^{n} \eta_{j k l}-\delta_{k}^{n} \eta_{l i j} \\
& \vartheta^{n} \wedge \eta_{k l i}=\delta_{i}^{n} \eta_{k l}+\delta_{l}^{n} \eta_{i k}+\delta_{k}^{n} \eta_{l i} \\
& \vartheta^{m} \wedge \eta_{k l}=\delta_{l}^{m} \eta_{k}-\delta_{k}^{m} \eta_{l} \\
& \vartheta^{j} \wedge \eta_{i}=\delta_{i}^{j} \eta
\end{aligned}
$$

The forms $\eta, \eta_{i}, \eta_{i j}$, and $\eta_{i j k}$ are Hodge dual to the forms $1, \vartheta^{i}, \vartheta^{i} \wedge \vartheta^{j}$, and $\vartheta^{i} \wedge \vartheta^{j} \wedge \vartheta^{k}$, respectively see Trautman [2]. 


\section{References}

1. Randono, A. Gauge Gravity: A forward-looking introduction. arXiv 2010, arXiv:1010.5822v1.

2. Trautman, A. Einstein-Cartan Theory. Symp. Math. 1973, 12, 139.

3. Wise, D.K. MacDowell-Mansouri Gravuty and Cartan Geometry. CQG 2010, 27, 155010. [CrossRef]

4. Hayashi, K.; Shirafuji, T. Gravity from Poincare Gauge Theory of the Fundamental Particles. Part V. Prog. Theor. Phys. 1981, 65, 525. [CrossRef]

5. Drechsler, W.; Mayer, M.E. Fiber Bundle Techniques in Gauge Theories. In Lectures Notes in Physics; Springer: Berlin/Heidelberg, Germany; New York, NY, USA, 1977; Volume 67.

6. Gürsey, F. Introduction to Group Theory. In Groups and Topology in Relativity; DeWitt, C., DeWitt, B., Eds.; Gordon and Breach: London, UK, 1964.

7. Wise, D.K. Symmetric Space Cartan Connections and Gravity in Three and Four Dimensions. arXiv 2009, arXiv:0904.1738v2.

8. Kobayashi, S.; Nomizu, K. Foundations of Differential Geometry; Interscience Publishers, a Division of John Wiley and Sons: New York, NY, USA; London, UK, 1963/1969.

9. Dubničkova, A. Topological Groups for Physicists; Dubna, Russia, 1987. (In Russian)

10. Kijowski, J. International Journal of Geometric Methods in Modern Physics; 2016; Volume 13, p. 1640008.

11. Schweizer, M.A. Gauge Theory and Gravitation. Ph.D. Thesis, University of Zurich, Zurich, Switzerland, 1980.

12. Sharpe, R.W. Differential Geometry. Cartan's Generalization of Klein's Erlangen Program; Springer: New York, NY, USA; Berlin/Heidelberg, Germany, 2000

13. Gancarzewicz, J. Foundations of Modern Differential Geometry; SCRIPT: Warsaw, Poland, 2010. (In Polish)

(C) 2019 by the authors. Licensee MDPI, Basel, Switzerland. This article is an open access article distributed under the terms and conditions of the Creative Commons Attribution (CC BY) license (http:/ / creativecommons.org/licenses/by/4.0/). 